\title{
Review
}

\section{Validation of analytical procedures by high-performance liquid chromatography for pharmaceutical analysis}

\author{
Masato Kazusaki, Shinji Ueda, Naoto Takeuchi, Yasutaka Ohgami \\ Investigational Drug Quality Assurance, Technology Research \& Development, Dainippon Sumitomo Pharma Co., Ltd. \\ 3-1-98 Kasugade-naka, Konohana-ku, Osaka 554-0022, Japan
}

\begin{abstract}
HPLC analysis is the critical factors in the drug developing process, and it is important to ensure the reliability of the analytical procedure to obtain meaningful data. The International Conference on Harmonization (ICH) has recognized the importance of validation concerning analytical procedures, and issued the guideline on Validation of Analytical Procedures (Q2) as a frame work for the validation study. This report complements the ICH guideline Q2, and provides practicable means for validation study focusing on the analyses by HPLC.
\end{abstract}

Keywords : validation, ICH, specificity, linearity, accuracy, precision

\section{Introduction}

Validation of analytical procedure is the process for proving that an analytical procedure is suitable for its intended purpose. Results obtained from method validation study can be used to judge the quality, reliability and consistency of analytical results.

Several articles have been published on the requirements of validation for analytical methods [1,2]. Green gave a practical guide for analytical method validation with a set of requirements for a method [3]. For the pharmaceutical industry, guidelines from the FDA [4-6] and US pharmacopoeia (USP) [7] provide a framework for performing validation study. Unfortunately, some of the definitions vary between the different organizations. To achieve harmonization for pharmaceutical applications, International Conference on Harmonization (ICH) was organized, and representatives from the pharmaceutical industry and regulatory agencies from the United States, Europe and Japan defined validation characteristics, requirements and methodology for analytical methods validation. For pharmaceutical analyses, an ICH guideline (Q2 (R1): Text on Validation of Analytical procedures and Methodology [8]) was issued for performing validation study. In this guideline, analytical procedures are classified into four categories. These four types of analytical procedures are: 1) identification tests, 2) quantitative tests for impurities, 3) limit tests for the control of impurities, 4) quantitative tests of the active moiety in bulk active pharmaceutical ingredient, formulated product, or other selected components in the formulated product. The assessment of validation characteristics should be based on the intended use of the method, and the level of stringency is proportional to the criticality of the analytical procedure in measurement. The ICH also recognizes that it is not always necessary to evaluate every validation characteristics. For identification test, only the validation characteristic of "specificity" should be established. Table 1 shows the two types of the analytical method for chromatographic analysis, such as assay method for measurement of the active moiety and impurity method for determination of target compounds at trace level, and validation characteristics to be investigated. For assay method, evaluation of detection limit (DL) and quantitation limit (QL) is not essential, because the target compound to be measured exists at high level. For quantitative analysis, a determination of DL is not necessary. There are no official guidelines on the sequence of validation experiments,

\section{Corresponding author: Masato Kazusaki}

Tel: +81-6-6439-8139

Fax: $+81-6-6466-5430$

E-mail address: masato-kazusaki@ds-pharma.co.jp 
Table 1. Validation characteristics for chromatographic analysis.

\begin{tabular}{|c|c|c|c|}
\hline Type of method & Assay method for the bulk active pharmaceutical ingredient & Impurity method for the & race compounds \\
\hline Type of analysis & Quantitative analysis by HPLC & Quantitative analysis by HPLC & Limit testing by TLC \\
\hline Specificity & + & + & + \\
\hline Detection limit & - & $+1-$ & + \\
\hline Quantitation limit & - & + & - \\
\hline Linearity & + & + & - \\
\hline Accuracy & + & + & - \\
\hline Precision & + & + & - \\
\hline Range & + & + & - \\
\hline
\end{tabular}

and the optimal sequence may depend on the analytical procedure. Based on the authors' experience, for conducting the validation of a liquid chromatographic method, the following sequence would be useful: specificity, detection limit and/or quantitation limit, linearity, accuracy, precision.

In the ICH Q2 guideline, validation characteristics to be investigated are all listed, but the acceptance limit for any items are not illustrated as an example. In addition to this, actual procedures to be conducted are not stated in detail. Vagueness in the ICH Q2 guideline necessitates effective protocol design. A well-designed experiment and statistically relevant approaches will facilitate the validation study on analytical procedure in accordance with the ICH guideline. This report describes approach for performing validation studies on the analytical procedure by HPLC. Acceptable criteria for each validation characteristics are also suggested in this report. Approaches described in this report would be applicable to other analytical techniques for biological samples and environmental analyses.

\section{Pre-validation requirements}

Chemicals, such as reagents and standards, should be available in sufficient quantities, accurately identified, sufficiently stable and checked for purity. Other materials and consumables, for example, chromatographic columns, should be qualified to meet the column's performance criteria. The validation experiments should also be carried out by an experienced analyst to avoid errors due to inexperience.

Validation on the analytical procedure should be performed with homogeneous samples, and validation data should be obtained by repeatedly analyzing aliquots of a homogeneous sample, each of which has been independently prepared according to the analytical method procedure.

\section{Analytical equipment qualification}

Satisfactory results of validation study can be obtained only with the equipment that operates well. For example, if detection limit is a critical factor for a specific method, the instrument's specification for baseline noise and the response to specified compounds should be verified.

Before undertaking the validation study, it is necessary to verify that the analytical system is adequately designed, maintained, qualified. ICH has published the guideline Q 7 [9] in which qualification of instruments are described to ensure the appropriateness of the analytical instruments. During the qualification stage of analytical instruments purchased from the vendor, installation qualification (IQ), operational qualification (OQ) and performance qualification (PQ) should be carried out. The IQ establishes that the instruments are well received as designed and specified, and that it is installed properly. The OQ ensures that modules of the HPLC system operates accurately and precisely according to the defined specifications concerning some parameters, such as the flow-rate for the pump, the injection volume for auto-sampler, temperature control for column oven, wavelength for UV-detector, etc. The PQ verifies the system performance. These steps are usually employed to verify that the system is adequate for the analysis to be performed.

\section{Stability of the analyte(s) in the solutions}

Some analytes in the solution might decompose prior to chromatographic investigations, for example, during the preparation of the sample solutions, extraction, cleanup or storage in the vials (in refrigerators or in an automatic sampler). To generate reproducible and reliable results, the stability of the analyte(s) in the solutions, and that of mobile phase must be determined prior to initiating the validation studies.

In many cases, samples are analyzed overnight using HPLC system equipped with the auto-sampler. For the assay method for bulk active pharmaceutical ingredient or the impurity method for trace compounds (impurities and contaminants), the analyte(s) in the sample solutions and the standard solution should be stable for 48 hours under the defined storage conditions. Mobile phases have to be stable for at least 48 hours. Acceptable stability criterion for the assay method is not more than $2.0 \%$ change in peak areas obtained from the stored solutions, relative to those from the solutions 
freshly prepared. As for the impurity method, acceptable stability criterion is not more than $10 \%$ change determined in the same way to the assay method. If the analyte(s) in the solutions are not stable at room temperature, then decreasing the storage temperature to 2$8^{\circ} \mathrm{C}$ may improve stability of the solutions. The mobile phase is considered to be stable if the stored mobile phase produces the equivalent chromatogram to that obtained with mobile phase freshly prepared. Assessment should be performed based on capacity factors, resolution and tailing factors.

\section{Protocol on analytical validation}

The protocol on the validation study should include the following points in the validation study: 1) the purpose and scope of the analytical method, 2) the type of analytical method and validation characteristics, 3) acceptance criteria for each validation characteristics. Consideration on the following points will be useful to prepare the protocol.

- What type of the samples will be measured by the analytical method? Will the samples be whole blood, serum, plasma, purified protein, chemicals? Are there interfering substances contained in the samples, if so, should they be detected or quantified?

-What is the expected concentration range?

- What level of specificity, detection limit or quantitation limit, linearity, accuracy and precision is required?

The purpose of answering the questions described above is to determine how best to meet the objective of the validation for analytical procedure. If the method is intended to quantitate the active pharmaceutical ingredient in the pharmaceuticals, or impurities at trace level, the method is categorized into the quantitative assay method, as shown in Table 1. If the method is intended to serve as a limit testing, the method is a qualitative method.

\section{Validation procedure}

In this section, we describe the meaning of the validation characteristics, and the actual approaches to perform the validation studies. An example of the acceptable criteria is also described.

\section{Specificity}

Specificity of the chromatographic analytical procedure is the ability to measure the analyte response in the presence of all potential sample components such as the starting materials, intermediates in the synthesis, and inactive ingredients in the formulated products, and the degradation products. Specificity in liquid chromatography is achieved by choosing optimal columns and setting chromatographic conditions, such as mobile phase composition, column temperature and detector wavelength. Besides chromatographic separation, the sample preparation procedure should also be optimized for best separation.

Specificity can be demonstrated by analyzing the samples containing impurities or other materials spiked onto the analyte(s) of interest. It is not necessary to spike potential interfering substances that do not reasonably exist in the testing samples. The degradation products could be generated by storing the analyte under the stress conditions sufficient to degrade it to approximately $90 \%$ purity. Typical stress conditions for generation of degradation products for bulk active pharmaceutical ingredients are heat $\left(50^{\circ} \mathrm{C}, 60^{\circ} \mathrm{C}\right)$, light (6500 lx of ultraviolet light), acidic condition (in $0.1 \mathrm{~mol} / \mathrm{L}$ hydrochloric acid solution), alkaline condition (in $0.1 \mathrm{~mol} / \mathrm{L}$ sodium hydroxide solution), and oxidant (in 3\% hydrogen peroxide solution). For formulated products, heat, light and humidity are the factors of severe conditions. Resulting mixtures should be analyzed, and the analyte peak is evaluated for peak purity and resolution from the nearest eluting peak. For biological analysis specificity studies should also be extended to assess interferences that may be caused by the components in urine, blood, etc. Optimized sample preparation can eliminate most of the matrix components.

In chromatographic analyses, it is difficult to ascertain whether the peaks in a chromatogram are pure, or consist of more than one compound. In the past, chromatographic parameters such as mobile phase composition were modified in order to investigate the peak purity. Recently the ultraviolet/visible diode-array detectors are being used. The level of impurities that can be detected with this instrument depends on the spectral difference, on the detector performance and on the software algorithm. Under ideal conditions, peak impurities at the level of $0.5 \%$ can be detected.

An example of specificity criteria for an impurity method for determining trace amounts of compounds is that the resolution factors are at least 1.2 among all the potential impurities that generated over the level of $0.1 \%$ in the stress conditions. For assay method, the resolution factor between target compound and impurities is at least 1.5. The desirable separation is shown in Figure 1.

Once acceptable resolution is achieved for the analyte and potential impurities, the chromatographic parameters, such as mobilephase composition, flow-rate, and detection mode, column type, should be considered to be set.

\section{Detection limit and quantitation limit}

The detection limit (DL) of an analytical procedure is the lowest analytical concentration at which an analyte(s) could be detected qualitatively. Typically peak heights are two or three times the noise level. The quantitation limit (QL) is also the lowest concentration at that level analyte can be quantitated with acceptable precision, requiring peak heights 10 to 20 times higher than the baseline noise. This signal-to-noise ratio is a good rule of thumb.

The ICH has recognized the signal-to-noise ratio is most con- 


\section{Analyte of interest for assay}

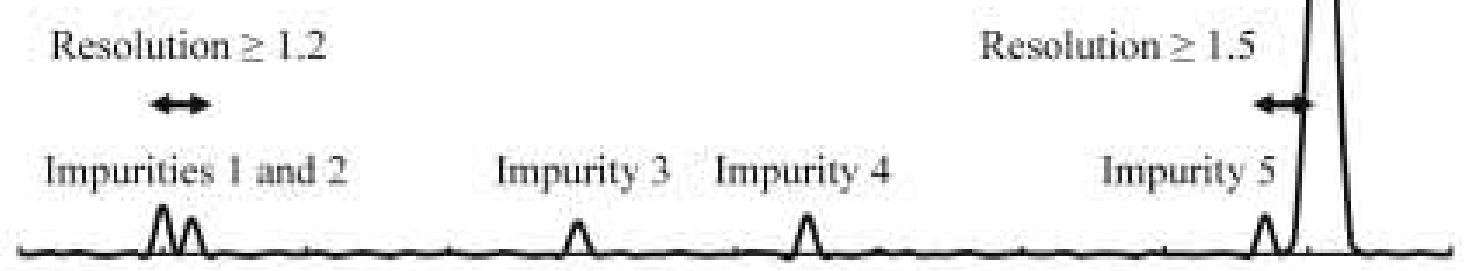

Figure 1. Desirable resolution between impurities and the target compound, and among impurities.

ventional, but also lists two other operations to determine DL and QL: visual non-instrumental method and a means of calculation. Visual non-instrumental methods may apply to a separation technique such as thin-layer chromatography. A means of calculation is based on the statistical background. Each method will give different results.

Currie proposed that DL should be decided exclusively based on error of the first kind $(\alpha)$ that is defined by the distribution of the blank noise [10]. He introduced the concept of critical level $\left(L_{C}\right)$ below which signals are judged not to be observed. Mathematically, the critical level is given as

$$
L_{C}=K_{\alpha} \sigma
$$

and the detection limit is expressed as below,

$$
D L=2 K_{\alpha} \sigma
$$

where $K_{\alpha}$ is the value concerning the standard normal distribution defining the probabilities, and $\sigma$ represents the standard deviation of the blank peaks. The standard deviation of the sample peaks at the DL level is assumed to be equal to that of the blank peaks. The two kinds of error should be considered: deciding that the substance is present when it is not ( $\alpha$; error of the first kind), and the converse, failing to decide that it is present when it exist ( $\beta$; error of the second kind). Generally, the acceptable value for $\alpha$ and $\beta$ are 0.05 in the pharmaceutical industries [11]. In this case, $K_{\alpha}$ is 1.65 , and DL is equal to $3.3 \sigma$. The relationship between $\mathrm{L}_{\mathrm{c}}, \mathrm{DL}$ and probability distributions is depicted in Figure 2.
In general, the physical quantity of interest (mass, concentration) is not directly measurable, but is calculated with the observed signal (peak area) through a calibration curve. DL is expressed in the following equation:

$$
D L=3.3 \times \frac{\sigma}{\text { slope }}
$$

where "slope" means that of the calibration curve. This equation could be converted into the following equation.

$$
\frac{\left(\frac{\sigma}{\text { slope }}\right)}{D L}=\frac{1}{3.3}=30 \%
$$

This equation means the relative standard deviation (RSD) at the detection limit level is $30 \%$. In the same manner, QL is also expressed in the following equation, and the RSD is $10 \%$ at the quantitation limit level.

$$
\begin{gathered}
Q L=10 \times \frac{\sigma}{\text { slope }} \\
\frac{\left(\frac{\sigma}{\text { slope }}\right)}{Q L}=\frac{1}{10}=10 \%
\end{gathered}
$$

Measurement of the magnitude of analytical background response is performed by analyzing an appropriate number of blank samples and calculating the standard deviation of these responses. The residual standard deviation or the standard deviation of $y$-intercepts of regression lines might be used as the standard deviation. 


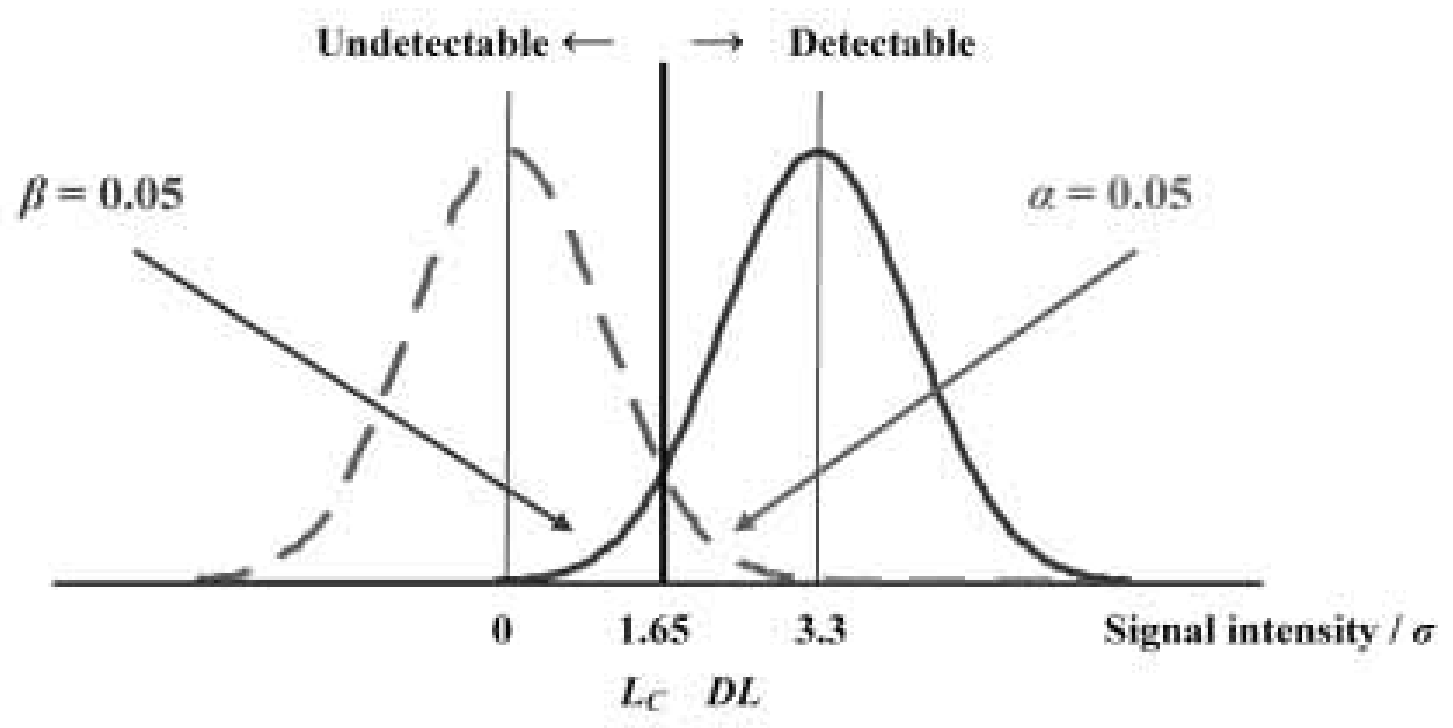

Figure 2. Relationship among critical level $\left(\mathrm{L}_{\mathrm{c}}\right)$, detection limit $(\mathrm{DL})$ and errors of the first and second kind.

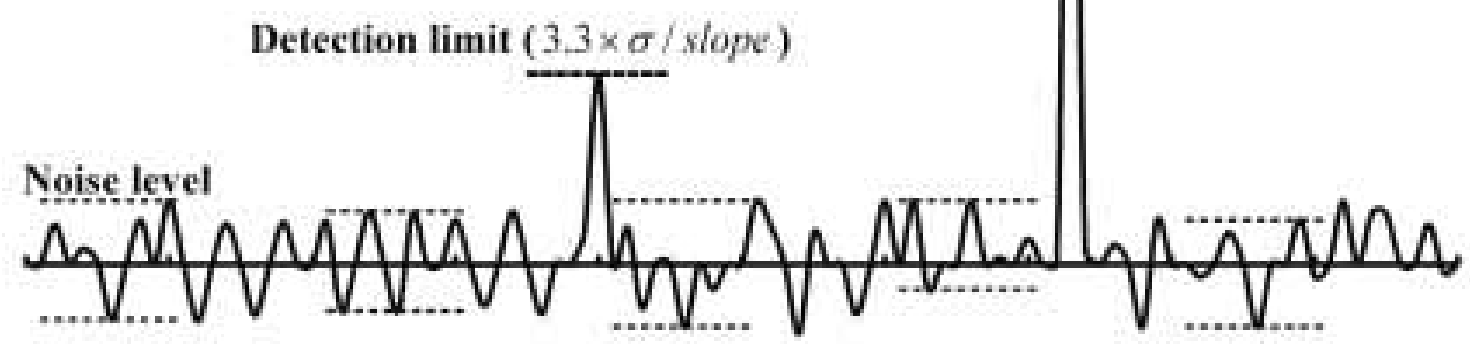

Figure 3. Typical chromatogram showing detection limit and quantitation limit. Noise levels vary, and are observed in the normal distribution pattern.

An example of criterion for detection limit is that, where the RSD of peak area of an impurity peak will be $\leq 30 \%$ when an analyte is analyzed in the short intervals. Similarly, criterion for quantitation limit is that RSD of the peak areas at that level are $\leq 10 \%$. Any estimated results of detection limit and quantitation limit must be verified with samples containing the corresponding analytes at DL or QL level, as shown in Figure 3.

Both DL and QL could be affected by the HPLC instruments. Sharper peaks result in a higher signal-to-noise ratio, resulting in lower DL and QL. We recommend verifying DL and QL when the HPLC system for routine analyses was changed, especially from the old HPLC system to the one of new type.

\section{Linearity}

Linearity of an analytical procedure is the ability for showing the response of the analyte is proportional to the analyte concentration within a given range. In practice, the linearity study should be designed to be appropriate for the intended analysis. At the completion of linearity studies, the appropriate concentration range would be set for all subsequent studies. For assay methods, linearity study is generally performed by preparing standard solutions at five concentration levels from 80 to $120 \%$ of the target analyte concentra- 
tion. For impurity methods, linearity is determined by preparing standard solutions at five concentration levels over a range from reporting threshold to $120 \%$ of the specification level. Reporting threshold is a limit above which an impurity in the bulk active pharmaceutical ingredient or formulated products should be reported to regulatory authorities, and specification level is a limit above which an impurity should not occur in the bulk active pharmaceutical ingredient or formulated products $[12,13]$. The ICH Q2 guideline specifies a minimum of five concentration levels along with certain minimum specified ranges, but do not require any proof of precision, because the linear relationship cannot be generated without sufficient precision.

Linearity is typically demonstrated via least-square regression. Acceptability of linearity data is often judged by examining the correlation coefficient and $y$-intercept, and residual sum of squares. For assay method, a correlation coefficient of more than 0.999 is generally considered as an evidence of acceptable fit of the data to the regression line. For impurity method, a correlation coefficient of more than 0.99 is generally acceptable. A linear regression equation applied to the results should have an intercept not significantly different from 0 . This result should be driven from the statistical assessment of calibration curve. It is also accepted that the $y$-intercept should be less than a few percent of the response obtained for the analyte at the target concentration. For example, the $y$-intercept for assay method should be less than $2.0 \%$ of the response of the analyte at the target concentration. The $y$-intercept for impurity method should be less than $10 \%$ of the response of the analyte at the specification level.

Linearity should also be evaluated graphically, in addition to mathematical evaluation described above. The evaluation is made by visually inspecting a plot of peak area as a function of analyte concentration, as shown in Figure 4. In addition to this approach, plots of the values obtained by the subtraction of the observed values from the predicted values (from the linear equation) against the concentration can help to assess the linearity. For linear ranges in the calibration curve, the deviations should be equally distributed between positive and negative values, as shown in Figure 5.

\section{Accuracy and precision}

Analytical results are obtained through the analytical procedure from the sample. In this case, analytical results involve two types of errors. One is the systematic error and the other is the random error. Systematic error is often caused from the analytical instruments, interference by the coexisting materials. Random error occurs whenever analyses are performed. These two types of errors in the analytical procedure should be investigated as validation characteristics of accuracy and precision.

Accuracy is the closeness of the analytical results obtained by the analyses to the true values, and usually presented as a percent of nominal. Accuracy in the absence of precision has little meaning. Accuracy is usually determined in one of the following four ways. First, accuracy can be assessed by analyzing a sample of known concentration (reference materials) and comparing the measured value to the true value. If National Institute of Standards

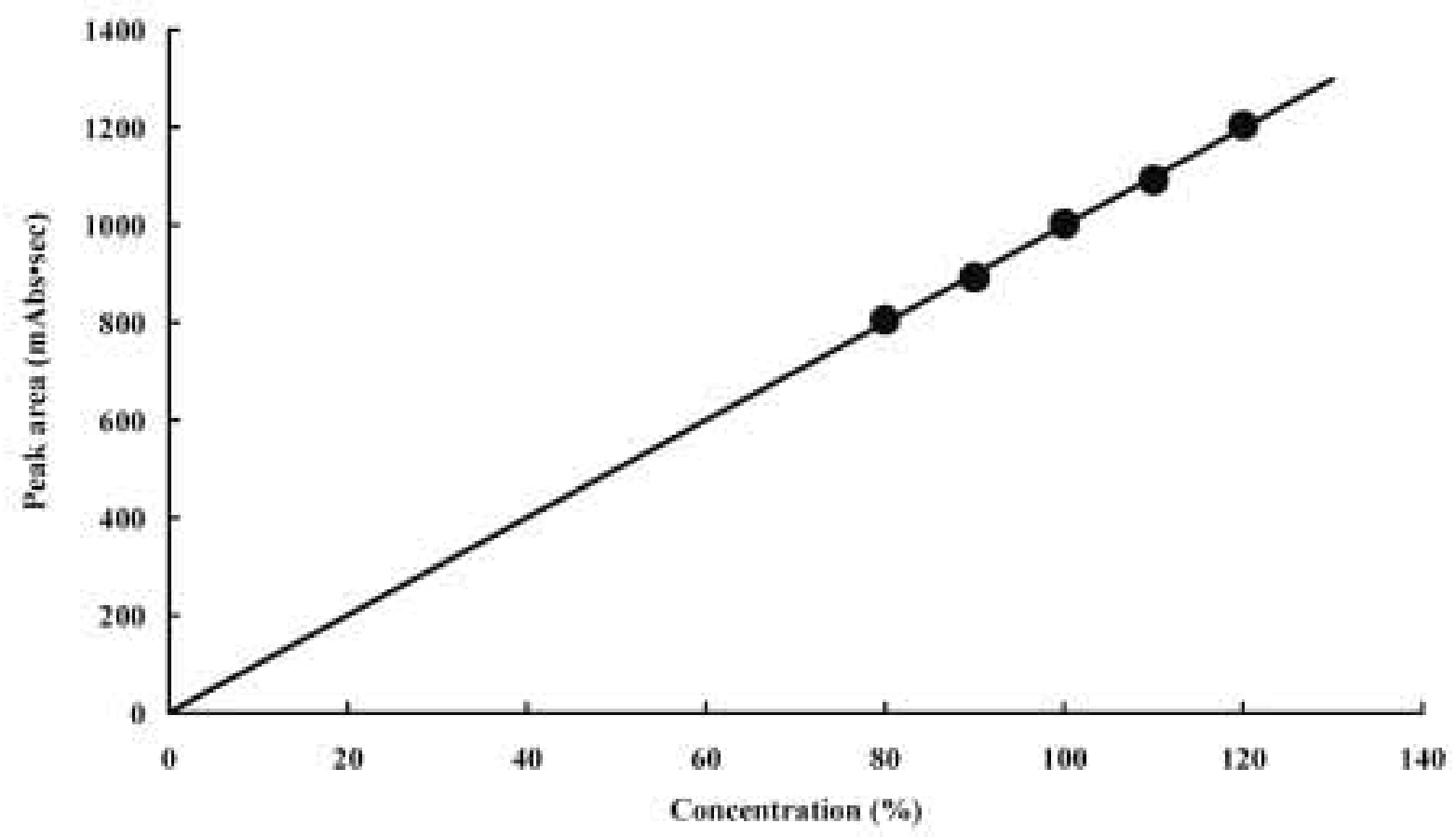

Figure 4. Typical calibration curve as a function of concentration and peak area. 


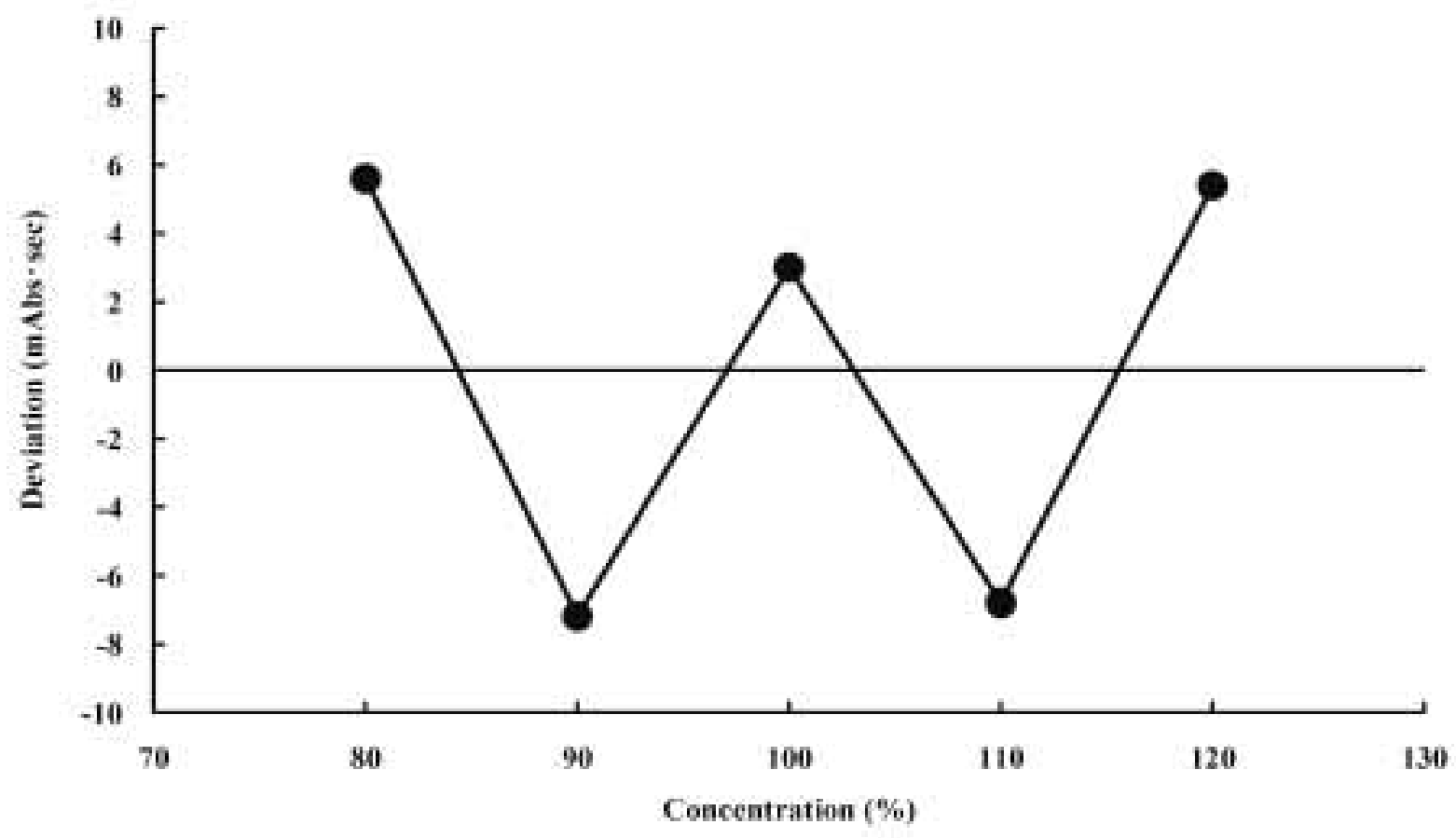

Figure 5. Deviation around the calibration line shown in Figure 5.

and Technology (NIST) standards could be available, those standards should be utilized. However, such a well-characterized standard could not be offered for new drug-related analytes. The second approach is to compare analytical results from the new analytical procedure with the results from an existing well-characterized procedure that is known to be accurate. Again, during the drug development stage in the pharmaceutical industries, such an alternate analytical procedure is usually not available. The third approach is performed by spiking analyte in blank matrices. Added amount corresponds to the true value. If potential impurities have been isolated, they would be added to the matrix to mimic impure samples. The analyte levels in the spiked samples should be determined using the same equation procedure as will be used in the defined analytical procedure.

The ICH Q2 guideline recommends accuracy to be assessed using a minimum of nine determinations over a minimum of three concentration levels covering the specified range. For assay methods, spiked samples are prepared in triplicate at three levels over a range of $80-120 \%$ of the target concentration. For impurity methods, spiked samples are prepared in triplicate over a range that covers the expected impurity content of the sample, such as reporting threshold to $120 \%$ of the specification level. After the calculation of the percent recovery, accuracy should be reported as percent recovery by the determination of known added amount of analyte in the sample or as the difference between the mean and the accepted true value, together with the confidence intervals. Confidence interval is calculated mainly from the average $(\bar{x})$, standard deviation
$(\sqrt{V})$. The average of the dataset is calculated using the following equation:

$$
\bar{x}=\frac{1}{n} \sum_{i=1}^{n} x i
$$

where $n$ means the number of samples measured in this study. The standard deviation is a measure of the spread of the values in the dataset, and can be calculated by the difference between the average and the individual values as follows.

$$
\sqrt{V}=\sqrt{\frac{\sum_{i=1}^{n}\left(x_{i}-\bar{x}\right)^{2}}{n-1}}
$$

Confidential intervals are used to indicate the reliability of an estimate. When the amount of pharmaceutical active ingredient in the formulated products is determined, the average value of the results is an estimate of an actual amount present in the formulated ones. A confidence interval provides limits around the mean values obtained through the assay procedure. In a confidence interval, the true value (population mean) lies with a given value of probability, usually $95 \%$. Confidence interval of population mean $(\mu)$ is expressed using the $t$-distribution:

$$
\bar{x}-t(\phi, \alpha) \frac{\sqrt{V}}{\sqrt{n}} \leq \mu \leq \bar{x}+t(\phi, \alpha) \frac{\sqrt{V}}{\sqrt{n}}
$$

where $\phi$ means degree of freedom, and $\alpha$ is error of the first kind. If $0 \%$ is out of the confidence interval of the accuracy (difference 
of the true value and observed value), analytical results are adversely affected by the systematic error. An example of accuracy criteria for impurity method is that the individual recoveries will be in the range of $80 \%$ to $120 \%$ at each concentration levels. For assay method that is applied to the formulated products, individual recovery will be from $95 \%$ to $105 \%$ at each concentration levels. For accuracy of the assay method for bulk active pharmaceutical ingredient, accuracy is estimated from the investigational results of specificity, linearity and precision.

The most important part of any validation study for analytical procedure is precision. The precision of an analytical method is the amount of variation in the results obtained from multiple analyses of the homogeneous samples. ICH guidelines break precision into three parts: repeatability, intermediate precision, and reproducibility.

Repeatability precision is expressed as the standard deviation of the analytical results when the analysis is carried out in a laboratory by an operator using an equipment over a relatively short time span. Repeatability is also termed intra-assay precision. The ICH guideline on methodology states two ways for data collection. One way is collecting data from a minimum of nine determinations (for example, three concentrations, three replicates each) over a minimum of three concentrations covering the target range. Another way is collecting data from at least 6 replications to be measured at 100 percent of the test target concentration. Precision data would be available from the triplicate analyses of spiked samples performed in the accuracy study. Documentation in support of precision (repeatability) studies should include the standard deviation and the confidence interval. Precision (repeatability) criteria of assay method for bulk active pharmaceutical ingredient are that the repeatability should be not more than $1.0 \%$, and that for formulated products not more than $2.0 \%$. For impurity method for determining tiny amount of compounds, these precisions should be not more than $10 \%$. Confidence interval of precision is also calculated using chi-square distribution,

$$
\sqrt{\frac{S}{x^{2}(\phi, \alpha / 2)}} \leq \sigma \leq \sqrt{\frac{S}{x^{2}(\phi, 1-\alpha / 2)}}
$$

where $S$ is sum of squared deviation, and obtained from the following calculation.

$$
S=\sum_{i=1}^{n}\left(x_{i}-\bar{x}\right)^{2}
$$

Intermediate precision is a term that has been defined by ICH as the long-term variability of the measurement process. Intermediate precision is the results from within-lab variations due to random events such as different day, different analysts, different analytical columns, different equipments, etc. The objective of intermediate precision validation is to verify that in the same laboratory the method will provide the same results. In determining intermediate precision, experimental design should be employed so that the effects (if any) of the individual variables can be monitored. The investigation consists of a minimum of two analysts on six different days with two replicates.

Reproducibility, which is determined by analyzing homogeneous samples in multiple laboratories, is often a part of inter-laboratory crossover studies. The objective is to verify that the method will provide the same results in different laboratories.

\section{Range}

The range of an analytical method is the interval between the upper and lower levels (including these levels) that have been demonstrated with precision, accuracy and linearity using the analytical method. So, the acceptable range will be defined as the concentration interval over which linearity, accuracy and precision are acceptable.

\section{Robustness}

The robustness of an analytical procedure is its ability to remain unaffected by small variation in the analytical parameters. The robustness is evaluated by varying the analytical parameters such as buffer $\mathrm{pH}$, flow rate, column temperature, injection volume, detection wavelength or mobile phase composition within a realistic range. The quantitative influence of the variables should be determined.

\section{Conclusion}

If the analysts in the pharmaceutical industry obtained the doubtful testing results through the invalid analytical procedure, they would realize that much amount of time should be required for solving problems. This kind of trouble would be avoided, provided that the validation study is performed properly. A well-defined validation process provides evidence that the system and method are suitable for its intended use. Performing a throughout validation study on an analytical procedure can be a tedious process. However, once validation studies are completed, the analysts can be confident in the ability of the analytical procedure to provide good quantitation.

We hope that we could provide a guide to help to understand how to perform a validation study on an analytical procedure that generates both useful and meaning data. This report focuses on performing a validation study for pharmaceuticals by HPLC system. This validation approach would be applied to the analytical methods using GC, HPLC, GC-MS, LC-MS for the biological samples or environmental pollution substances. Many of the principles, separation techniques, and requirements are common to all types of 
chromatographic analytical methodologies.

\section{References}

[1] Inman, E. L.; Frischmann, J. K.; Jimenez, P. J.; Winkle, G. D.; Persinger, M. L.; Rutherford, B. S. J. Chromatogr. Sci. 1987, 25, 252-256.

[2] Shah, V. P.; Midha, K. K.; Dighe, S.; McGilverray, I. J.; Skey, J. P.; Yacobi, A.; Layoff, T.; Viswanathan, C. T.; Cook, C. E.; McDowall, R. D.; Pittman, K. A.; Spector, S. Pharma. Res. 1992, 9, 588-592.

[3] Green, J. M. Anal. Chem. 1996, 305A-309A.

[4] U.S. Department of Health and Human Services, Food and Drug Administration, Center for Drug Evaluation and Research (CDER), Center for Veterinary Medicine (CVM). Guidance for Industry, Bioanalytical Method Validation, 2001.

[5] U.S. Department of Health and Human Services, Food and Drug Administration, Center for Drug Evaluation and Research (CDER). Reviewer Guidance, Validation of chromatographic methods, 1994.

[6] U.S. Department of Health and Human Services, Food and Drug Administration, Center for Drug Evaluation and Research (CDER), Center for Biologics Evaluation and Re- search (CBER). Guidance for Industry, Analytical Procedures and Methods Validation, 2000.

[7] United States Pharmacopoeia 30; Validation of compendial methods, section $<1225>$.

[8] International Conference on Harmonization of Technical Requirements for Registration of Pharmaceuticals for Human Use. Validation of Analytical Procedures: Text and Methodology, Q2 (R1), 2005.

[9] International Conference on Harmonization of Technical Requirements for Registration of Pharmaceuticals for Human Use. Good Manufacturing Practice Guide for Active Pharmaceutical Ingredients, Q7, 2000.

[10] Currie, L. A. Anal. Chem. 1968, 40, 586-593.

[11] The Ministry of Health, Labor and Welfare, Validation of analytical procedures. The Japanese Pharmacopoeia, Sixteenth edition, 2011.

[12] International Conference on Harmonization of Technical Requirements for Registration of Pharmaceuticals for Human Use. Impurities in New Drug Substances, Q3A (R2), 2006.

[13] International Conference on Harmonization of Technical Requirements for Registration of Pharmaceuticals for Human Use. Impurities in New Drug Products, Q3B (R2), 2006. 